\title{
Research of Ofloxacin Based on Terahertz Spectrum
}

\author{
Song Zhang ${ }^{1, a}$, Xiai Chen ${ }^{1,2,3, b, ~ *}$, Xiaohong Gu ${ }^{1, c}$, Qiang Wang ${ }^{1, d}$, Ling Wang ${ }^{1, e}$ \\ ${ }^{1}$ School of Mechanical and Electrical Engineering, China Jiliang University, Hangzhou, China \\ ${ }^{2}$ Zhejiang Yalong Educational Equipment Joint-Stock Co., Ltd, Wenzhou, China \\ ${ }^{3}$ State Key Lab. of Industrial Control Technology, Department of Control Science and \\ Engineering, Zhejiang University, Hangzhou,China \\ azsong16zj@163.com, bxachen@cjlu.edu.cn, '06a0102081@cjlu.edu.cn, dqiangwang@cjlu.edu.cn, \\ ewangling@cjlu.edu.cn
}

Keywords: Terahertz time-domain spectroscopy; Absorption spectrum; Density functional theory; Ofloxacin

\begin{abstract}
The absorption coefficient, refractive index and optical fingerprint characteristics were acquired by terahertz time-domain spectroscopy in the range of $0.4 \sim 1.5 \mathrm{THz}$ of the capsule drug ofloxacin. The results show that ofloxacin had multiple absorption peaks with different intensities within the frequency range, and the absorption coefficient spectrum could be used for the identification of ofloxacin. In the range of $0.4 \sim 1.5 \mathrm{THz}$, the absorption spectra of ofloxacin molecules were simulated by Gaussion software. The vibration modes of some absorption peaks in the absorption spectra were analyzed and identified using the density functional theory. At 1.06 and $1.13 \mathrm{THz}$, the experimental results show that the absorption peaks are consistent with the absorption peaks of 1.07 and $1.18 \mathrm{THz}$ in the theoretical spectrum, which are caused by the vibrational modes in the molecule ofloxacin.
\end{abstract}

\section{Introduction}

Terahertz radiation is a generic term for electromagnetic radiation in a particular band. Its band is located between the microwave and infrared, the frequency range of $0.1 \sim 10 \mathrm{THz}$, and it is also named as the far infrared ray in the field of spectroscopy [1].

Capsules are high-grade plastic products which are made from animal skin, bone and tendon, after a complex physical and chemical treatment. Because of the low photon energy, the terahertz wave will not cause ionization or other damage to biomolecules, it is the ideal source for biomedical testing [2].

Ofloxacin belonging to the third generation quinolone antibacterial drugs is the main component of medical capsules, which is the treatment of the respiratory tract, throat and other parts caused by Gram negative bacteria, it can hinder Staphylococcus, Streptococcus pneumoniae and Escherichia coli. These analytical methods often have shortcomings such as high equipment requirements, long identification time and cumbersome operation methods, which are not conducive to the rapid identification of ofloxacin [3].

Terahertz time-domain spectroscopy(THz-TDS) can be used to detect the THz-TDS information, the optical parameters of the material can be obtained by optical parametric model, after that the physical and chemical information of the substance can be determined [4] [5]. The preparation process of the sample is simple and convenient, and it is necessary to carry out powder pressing. The whole process is fast and accurate, and it is expected to become a new material of rapid detection technology. 


\section{Experimental preparation.}

With a molecular formula of C18H20FN3O4, ofloxacin has some aliases, including Hoe-28o, TV-10o. As capsules, the content of ofloxacin is white to pale yellow powder, and the chemical structure of it is shown in Fig.1.<smiles>CC1COc2c(N3CCN(C)CC3)c(F)cc3c(=O)c(C(=O)O)cn1c23</smiles>

Fig.1.Molecular structure of ofloxacin

Ofloxacin pure purchased from Dalian Meilune Biotechnology Co., Ltd., purity> 99\%. The powder adhesion of pure ofloxacin is poor, so polyethylene powder in the $\mathrm{THz}$ band without absorption peak is selected as a binder. The mixture of ofloxacin and polyethylene was 1: 1 by mass, grinded using an agate bowl after the mixture was dried. The mixture was pressed into a sample which was smooth and crack-free and parallel at both ends with diameter of $13 \mathrm{~mm}$ and thickness of 1.05mm, using a FW-4 tableting machine (Tianjin Tianguang Optical Instrument Co., Ltd.).

The THz-TDS experimental system used in this paper is Model Z-3 series developed by Zomega Terahertz Corporation. This system consists of femtosecond laser, $\mathrm{THz}$ pulse detection optical path, $\mathrm{THz}$ pulse generation optical path and $\mathrm{THz}$ data calculation. The laboratory temperature is $22.1{ }^{\circ} \mathrm{C}$, and the dehumidification treatment is carried out with nitrogen, so that the relative humidity of the sample chamber is kept at zero.

\section{Experimental Spectra and Theoretical Spectrum of Ofloxacin.}

Fig.2 shows the time-domain spectra of ofloxacin and reference, it can be clearly seen from the figure that the sample signal has a time delay with respect to the reference signal, because the refractive index of the sample in the THz pulse is greater than the refractive index in nitrogen [6].

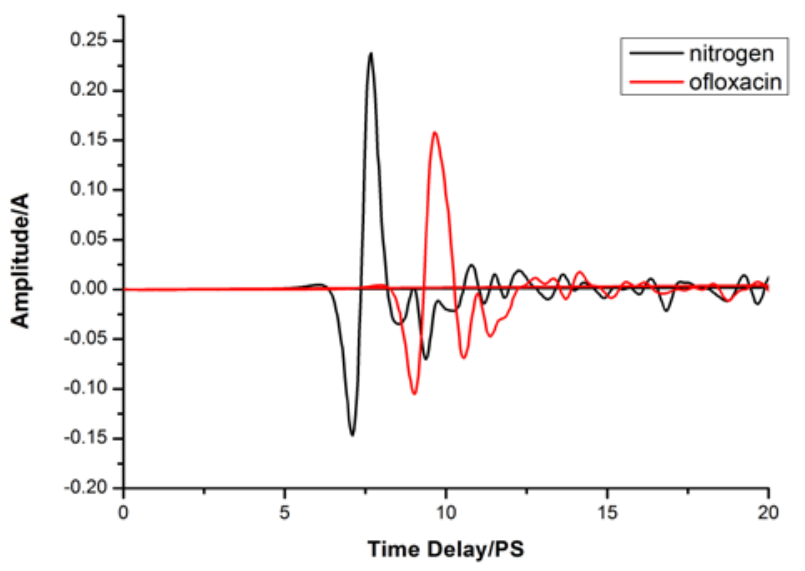

Fig.2.Time-domain spectra of the nitrogen and ofloxacin

In the experiment, the molecular structure of ofloxacin was drawn by ChemDraw Ultra, the structure optimization of ofloxacin was carried out by B3LYP algorithm and 6-31G (d) basis, in order to calculate the vibration frequency in later period. Then the vibration frequency structure information of ofloxacin was obtained by Gaussian 03 software. The dynamic vibration of ofloxacin in $\mathrm{THz}$ band was observed by Gaussian View software [7] [8]. Experimental results do not appear virtual frequency phenomenon, consistent with the actual situation. 


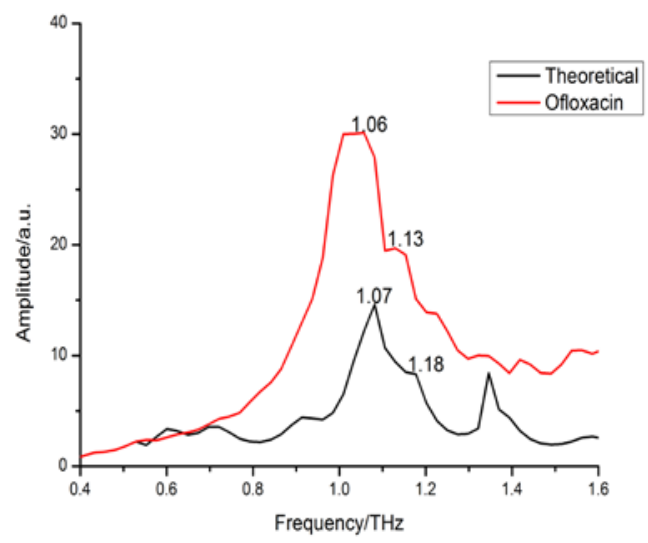

Fig.3. Comparison between absorption coefficient experimental and theoretical spectra of ofloxacin

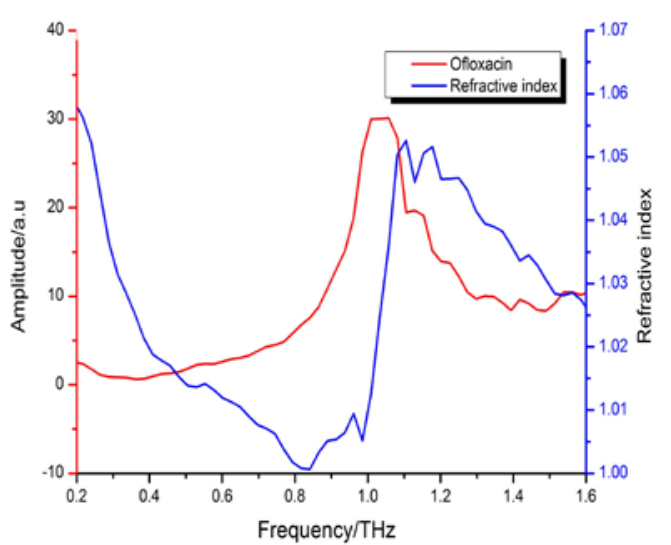

Fig.4. Refractive index spectra and absorption coefficient spectra of ofloxacin

Fig. 3 shows that in the $0.4 \sim 1.5 \mathrm{THz}$ band, there are two theoretical absorption peaks of ofloxacin, 1.07 and $1.18 \mathrm{THz}$, and these peaks are basically consistent with the experimental result -1.06 and 1.13THz.

According to Fig. 4, the refractive index of ofloxacin in the range of $0.4 \sim 1.5 \mathrm{THz}$ corresponds to the characteristic absorption peak in the absorption spectrum, which is consistent with the Kromers-Kroning relationship [9]. It can be seen clearly from Fig. 4 that there are obvious absorption peaks at 1.06 and $1.13 \mathrm{THz}$ of ofloxacin.

\section{Absorption peak analysis.}

The partial $\mathrm{THz}$ absorption peaks of ofloxacin in the range of $0.4 \sim 1.5 \mathrm{THz}$ were analyzed and identified by Gaussian software.

The absorption at $1.07 \mathrm{THz}$ is the out-of-plane reverse swing of the carboxyl group $25 \mathrm{O}=22 \mathrm{C}-25 \mathrm{O}-27 \mathrm{H}$ with the $22 \mathrm{C}-20 \mathrm{C}$ bond axis, and the out-of-plane rocking vibration of the $15 \mathrm{C}$ methyl group with the $13 \mathrm{~N}-15 \mathrm{C}$, these two torsional oscillations drive the torsional pendulum vibration of the entire ofloxacin molecular skeleton, as shown in Fig.5.

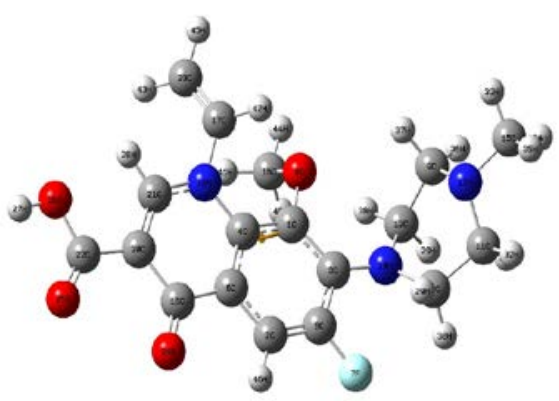

Fig.5.Vibration mode of ofloxacin at 1.07THz Fig.6.Vibration mode of ofloxacin at $1.18 \mathrm{THz}$

Fig.6 shows there is an absorption peak at $1.18 \mathrm{THz}$, the absorption peak was generated by the $19 \mathrm{C}=24 \mathrm{O}$ bond around the nitrogen-containing heterocyclic ring to do outside the rocking vibration, and the out-of-plane deformation vibrations of the carboxyl group $25 \mathrm{O}=22 \mathrm{C}-25 \mathrm{O}-27 \mathrm{H}$, and the 14N-10C- 9C-12C deformation twist.

The theoretical model of the Gaussion software simulates various vibrational modes within the solute of the substance, the absorption peaks at 1.06 and $1.13 \mathrm{THz}$ in the experimental spectra are caused by the vibration modes in the molecule of ofloxacin, which correspond to the absorption peaks 
at 1.07 and $1.18 \mathrm{THz}$ in the theoretical spectrum, respectively. And the shift of the absorption peak position is mainly due to the temperature and the solid phase of the sample caused by the material.

\section{Conclusions}

The optical parameters such as absorption coefficient and refractive index of ofloxacin in the terahertz band were measured, and the fingerprint of ofloxacin was obtained. In addition, the theoretical absorption spectra of ofloxacin molecules have been simulated by density functional theory and compared with the absorption spectra obtained by experiments. The main absorption peaks of ofloxacin were analyzed and identified, the results show that the absorption peaks were caused by the internal vibration modes. Based on the above studies, the technique of terahertz time-domain spectroscopy can be extended to the analysis of various capsules, and the technology is expected to be applied in the field of capsule drug detection.

\section{Acknowledgements}

This research work is supported by National Natural Science Foundation of China (Grant No. 51504228), Natural Science Foundation of Zhejiang province of China (Grant No. LQ14F050003), Natural Science Foundation of Zhejiang province of China (Grant No. LY14F030019) , Natural Science Foundation of Zhejiang province of China (Grant No. LY14E040002) and 2016 postdoctoral research projects of Zhejiang Province of China.

\section{References}

[1] XU JING-ZHOU, ZHANG Xi-CHENG. Terahertz Science Technology and Application. Beijing: Peking University Press, 2007.

[2] Portnoi M E, Kibis O V, Costa M R D. Terahertz applications of carbon nanotubes[J]. Superlattices \& Microstructures, 2015, 43(5-6):399-407.

[3] Qin W, Zhang J, Yue Z, et al. Adsorption and release of ofloxacin from acid- and heat-treated halloysite[J]. Colloids \& Surfaces B Biointerfaces, 2014, 113(1):51-58.

[4] Harsha S S, Grischkowsky D. Terahertz (far-infrared) characterization of tris (hydroxymethyl) aminomethane using high-resolution waveguide THz-TDS[J]. Journal of Physical Chemistry A, 2010, 114(10): 3489-94.

[5] Mehdi Hosseini. Optimization of the THz radiation from superconductor at non-laminar regime[J]. Physica C: Superconductivity and its Applications, 2016, 529:36-39.

[6] KANG Xu-sheng, HOU Di-bo, ZHANG Guang-xin, CHEN Xi-ai, YUE Fei-heng, HUANG Ping-jie, ZHOU Ze-kui. Spectral Analysis of Nitrofurantoin in the Terahertz Frequency Range[J]. Spectroscopy and Spectral Analysis, 2012, 32(7):1744-1747.

[7] Woods K N. THz time scale structural rearrangements and binding modes in lysozyme-ligand interactions[J]. Journal of Biological Physics, 2014, 40(2):121-37.

[8] Hua Y F, Zhang H J. Qualitative and Quantitative Detection of Pesticides with Terahertz Time-Domain Spectroscopy[J]. IEEE Transactions on Microwave Theory and Techniques, 2010, 58(7): 2064-2070.

[9] Peiponen K E, Saarinen J J. Generalized Kramers-Kronig relations in nonlinear optical- and THz-spectroscopy[J]. Reports on Progress in Physics, 2009, 72(5): 56401-56419. 